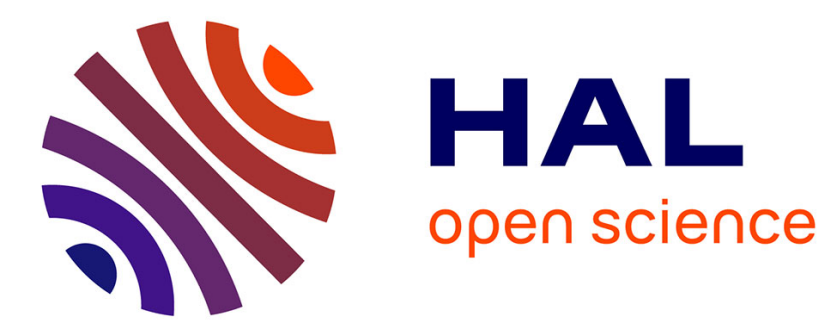

\title{
Driver steering assistance for lane departure avoidance
}

\author{
N. Minoiu-Enache, M. Netto, S. Mammar, B. Lusetti
}

\section{To cite this version:}

N. Minoiu-Enache, M. Netto, S. Mammar, B. Lusetti. Driver steering assistance for lane departure avoidance. Control Engineering Practice, 2009, vol17, n6, p642-51. hal-00506570

\section{HAL Id: hal-00506570 \\ https://hal.science/hal-00506570}

Submitted on 28 Jul 2010

HAL is a multi-disciplinary open access archive for the deposit and dissemination of scientific research documents, whether they are published or not. The documents may come from teaching and research institutions in France or abroad, or from public or private research centers.
L'archive ouverte pluridisciplinaire HAL, est destinée au dépôt et à la diffusion de documents scientifiques de niveau recherche, publiés ou non, émanant des établissements d'enseignement et de recherche français ou étrangers, des laboratoires publics ou privés. 


\title{
Driver steering assistance for lane departure
}

\section{avoidance}

\author{
N. Minoiu Enache ${ }^{\mathrm{a}, *, 1}$, M. Netto ${ }^{\text {a }}, \mathrm{S}$. Mammar $^{\mathrm{b}}$, B. Lusetti ${ }^{\mathrm{a}}$ \\ ${ }^{\mathrm{a}}$ LCPC/INRETS - LIVIC Laboratoire sur les Interactions \\ Véhicules-Infrastructure-Conducteurs, 14 route de la minière, 78000 Versailles, France \\ ' IBISC/CNRS-FRE 3190, Université d'Evry Val d'Essonne, 40 Rue du Pelvoux, CE 1455, \\ 91025, Evry, Cedex, France
}

\begin{abstract}
In this paper, a steering assistance system is designed and experimentally tested on a prototype passenger vehicle. Its main goal is to avoid lane departures when the driver has a lapse of attention. Based on a concept linking Lyapunov theory with Linear Matrix Inequalities (LMI) optimization, the following important features are ensured during the assistance intervention: the vehicle remains within the lane borders while converging towards the centerline, and the torque control input and the vehicle dynamics are limited to safe values to ensure the passengers' comfort. Because the steering assistance takes action only if necessary, two activation strategies have been proposed. Both activation strategies were tested on the prototype vehicle and were assessed as appropriate. However, the second strategy showed better reactivity in case of rapid drifting out of the lane.
\end{abstract}

Key words: active safety, lane departure avoidance, lateral vehicle control, Lyapunov function, LMI, switched system 


\section{Introduction}

The failure of a driver to remain in the correct lane due to inattention, illness, or sleepiness is one of the most important causes of accidents. NHTSA (2006) estimated that running off the road caused about $28 \%$ of the fatal motor vehicle crashes in the US in 2005. Moreover, drowsy, sleeping, or fatigued drivers and inattentive drivers caused about $2.6 \%$ and $5.8 \%$ of the fatal crashes, respectively.

In order to prevent this type of accident, vehicles have increasingly been equipped with electronic control systems that provide active safety (Isermann (2008)). New steering assistance systems have been developed both to decrease the driver's workload and to prevent lane departures (Shimakage et al. (2002)), (Rossetter et al. (2004)), (LeBlanc et al. (1996)) and (Nagai et al. (2002)). Eidehall et al. (2007) proposed an integrated road geometry estimation method using vehicle tracking to improve the activation accuracy of an emergency lane assist system.

The work presented in this paper aims at developing a steering assistance system that helps the driver guide the vehicle to the center of the lane during diminished driving capability due to inattention, fatigue, or illness. For vehicles equipped with a conventional steering column, an important issue in implementing a steering assistance system arises: How to introduce automation to help the driver simultaneously with his own actions on the steering wheel? Any intrusion by an automatic steering system might be immediately felt by the driver on the steering wheel. Re-

\footnotetext{
* Corresponding author.

Email addresses: nicoleta.minoiu@lcpc.fr (N. Minoiu Enache),

mariana.netto@lcpc.fr (M. Netto), said.mammar@iup.univ-evry.fr (S.

Mammar), benoit.lusetti@lcpc.fr (B. Lusetti).

1 Tel. +33-(0)1-40432919; fax +33-(0)1-40432930.
} 
ciprocally, any torque imposed on the steering wheel by the driver could be considered by the automatic control system as a disturbance input.

To overcome this difficulty, a switching strategy coupled with a designed lateral control law is proposed in this work. The switching strategy assigns the steering control either to the driver or to the steering assistance system. More specifically, the steering assistance system has been developed to take over the driver's action when it is determined that he has lost attention and to return control of the vehicle upon the driver's request when the vehicle is out of danger.

The main contributions of this paper are:

(1) A theoretical framework for handling the interactions between the driver and the steering assistance system that ensures bounded dynamics of the switched system.

(2) Guaranteed bounds for the displacement of the front wheels with respect to the center of the lane, as well as a control torque input that is limited by the design method during the assistance intervention.

(3) Validation of the theoretical results by experimental tests using a prototype vehicle.

The contents of this paper are organized as follows. Section 2 presents the vehicle model, including the electrically powered steering column. The specifications of the steering assistance system are given in Section 3.1, while Sections 3.2 and 3.3 address the definition of a "normal driving" situation and the design of the switching strategy. Sections 4 and 5 describe the design of the steering control law, and subsequently the evolution of the trajectories of the switched system. Section 6 contains the results of the practical implementation of the steering assistance system under the first switching strategy. Following these results, new activation rules for 
a second switching strategy are developed and practically implemented in Section 7. Conclusions are presented in Section 8.

\section{Vehicle model with electrically powered steering}

Since this study is focused on the lateral control of a vehicle, a classical fourth order linear model (“bicycle model”, Fig. 1) was used (Ackermann et al. (1995)). The effect of road curvature was neglected and the road was assumed to be straight, which is realistic for the highway driving environment addressed in this work. The steering torque necessary for the assistance is provided by a DC motor mounted on the steering column, for which a second order model was adopted. The vehicle model, including the electrical steering assistance model, is given by:

$$
\begin{gathered}
\dot{\mathbf{x}}=\mathbf{A} \cdot \mathbf{x}+\mathbf{B} \cdot\left(T_{a}+T_{d}\right), \\
\mathbf{A}=\left(\begin{array}{cccccc}
a_{11} & a_{12} & 0 & 0 & b_{1} & 0 \\
a_{21} & a_{22} & 0 & 0 & b_{2} & 0 \\
0 & 1 & 0 & 0 & 0 & 0 \\
v & l_{S} & v & 0 & 0 & 0 \\
0 & 0 & 0 & 0 & 0 & 1 \\
\frac{T_{S_{\beta}}}{I_{S} R_{S}} & \frac{T_{S_{r}}}{I_{S} R_{S}} & 0 & 0 & -\frac{2 K_{p} c_{f} \eta_{t}}{I_{S} R_{S}^{2}} & -\frac{B_{S}}{I_{S}}
\end{array}\right),
\end{gathered}
$$




$$
\mathbf{B}=\left(0,0,0,0,0, \frac{1}{R_{S} I_{S}}\right)^{T}
$$

where

$$
\begin{array}{cc}
a_{11}=-\frac{2\left(c_{r}+c_{f}\right)}{m v}, & a_{12}=-1+\frac{2\left(l_{r} c_{r}-l_{f} c_{f}\right)}{m v^{2}}, \\
a_{21}=\frac{2\left(l_{r} c_{r}-l_{f} c_{f}\right)}{J}, & a_{22}=-\frac{2\left(l_{r}^{2} c_{r}+l_{f}^{2} c_{f}\right)}{J v}, \\
c_{r}=c_{r 0} \mu, & c_{f}=c_{f 0} \mu, \\
b_{1}=\frac{2 c_{f}}{m v}, & b_{2}=\frac{2 c_{f} l_{f}}{J}, \\
T_{S \beta}=\frac{2 K_{p} c_{f} \eta_{t}}{R_{S}}, & T_{S_{r}}=\frac{2 K_{p} c_{f} l_{f} \eta_{t}}{R_{S} v} .
\end{array}
$$

The definitions and the numerical values of the above parameters are given in Table 2 at the end of the paper. The state vector is $\mathbf{x} \triangleq\left(\beta, r, \psi_{L}, y_{L}, \delta_{f}, \dot{\delta}_{f}\right)^{T}$, where $\beta$ denotes the side slip angle, $r$ is the yaw rate, $\psi_{L}$ is the relative yaw angle, $y_{L}$ is the lateral offset with respect to the lane centerline at a look-ahead distance $l_{S}, \delta_{f}$ is the steering angle, and $\dot{\delta}_{f}$ is its derivative. The inputs for the system given in Eq. (1) are the driver's torque $T_{d}$ and the assistance torque $T_{a}$. The whole state vector is considered to be available for measurement.

Remark 1 It can easily be shown that the system given in Eq. (1) is controllable except for a longitudinal speed $v$ equal to zero. The matrix $\mathbf{A}$ has two poles at the origin, indicating instability of the linear system. 


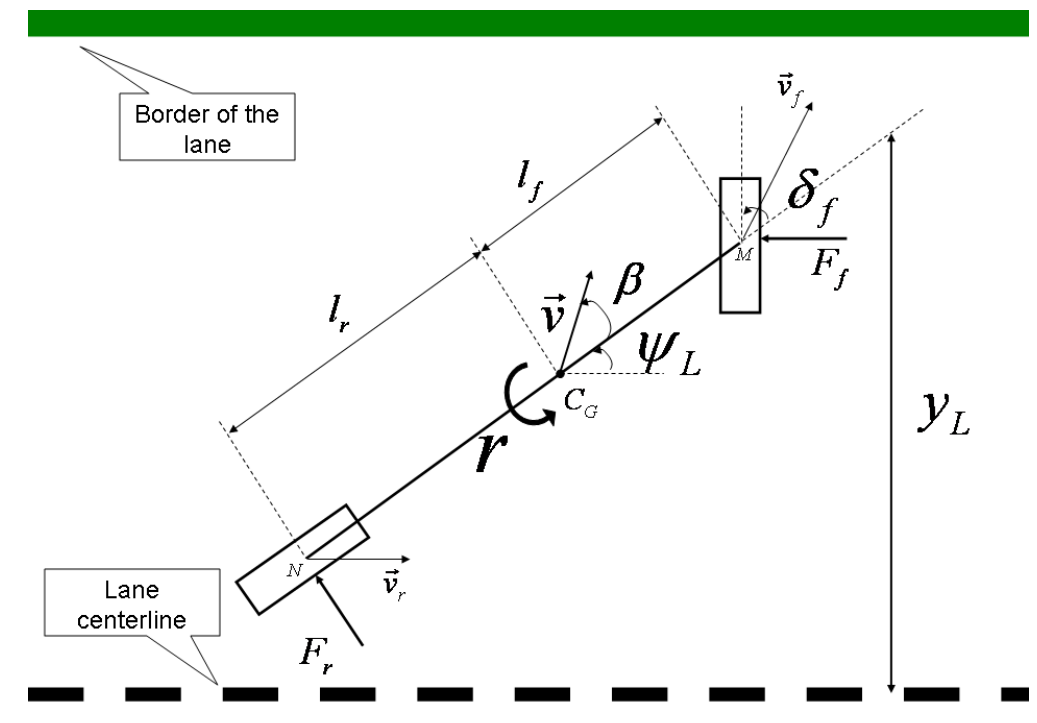

Fig. 1. Vehicle "bicycle" model.

\section{Steering assistance requirements and problem formulation}

\subsection{Steering assistance requirements}

The proposed steering assistance system aims at avoiding unintended lane departures during "normal driving". "Normal driving" is defined as a driving situation during which the driver is following the center of the lane without performing any special maneuver (e.g., overtaking, cornering, change of direction). The steering assistance system should accomplish its task by means of two intelligent modules:

(1) A switching strategy module that activates and deactivates the steering assistance system depending on the driver's attention and on the danger of lane departure.

(2) A second module that contains a steering control law should drive the vehicle during the driver's inattention. The steering control law should satisfy the following requirements: (a) the closed loop system vehicle-steering control law 


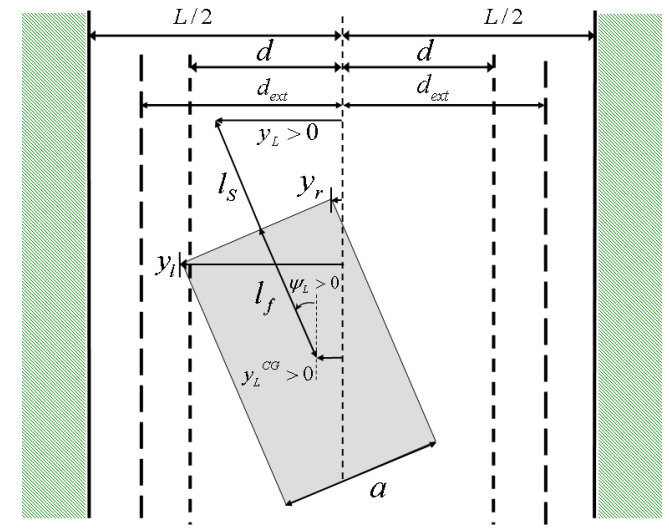

Fig. 2. Vehicle in the lane.

has to be asymptotically stable to zero steady state, (b) the vehicle shall not cross the lane borders during the assistance intervention period, (c) moreover, the overshoot of the front wheels with respect to a fixed predefined center lane strip has to be as small as possible, and (d) the vehicle state variables and the steering assistance torque have to be bounded to guarantee safety and comfort.

\subsection{Mathematical definition of the "normal driving" zone}

First of all, the qualitative description of the "normal driving" situation has to be transposed into a formal mathematical description. Such a driving situation can been characterized by the positions of the vehicle's front wheels, which are generally confined to a strip along the center of the lane during a normal lane keeping maneuver. This center strip is assumed to have a width of $2 d$, where the total lane width is $L$ and $2 d<L$, as shown in Fig. 2. On the other hand, during "normal driving", the vehicle state variables are assumed to have a limited range, bounded within a region in the state space. This region was defined by the maximum absolute values: $|\beta| \leq \beta^{N},|r| \leq r^{N},\left|\psi_{L}\right| \leq \psi_{L}^{N},\left|y_{L}\right| \leq y_{L}^{N},\left|\delta_{f}\right| \leq \delta_{f}^{N}$ and $\left|\dot{\delta}_{f}\right| \leq \dot{\delta}_{f}^{N}$.

The coordinates of the two front wheels $y_{l}$ and $y_{r}$ are calculated with respect to 
the center of the lane from the geometrical model in Fig. 2. The relative position of the vehicle with respect to the lane, represented in Fig. 2, is characterized by a relative yaw angle $\psi_{L}$, a lateral offset from the centerline $y_{L}^{C G}$ at the vehicle center of gravity, and a lateral offset $y_{L}$ measured at a look-ahead distance $l_{S}{ }^{2}$. Assuming a small relative yaw angle $\psi_{L}$, the following equalities can be written:

$$
\begin{aligned}
& y_{l}=y_{L}^{C G}+l_{f} \psi_{L}+\frac{a}{2}, \\
& y_{r}=y_{L}^{C G}+l_{f} \psi_{L}-\frac{a}{2},
\end{aligned}
$$

where $l_{f}$ is the distance from the vehicle center of gravity to the front axle and $a$ is the vehicle width ${ }^{3}$. Generally, the vehicle road sensing system used for lateral control is limited to a camera mounted with a frontal view and image processing algorithms to measure the lateral offset and the relative yaw angle. For this reason, for a straight road and for a small angle $\psi_{L}$, the following equality containing $y_{L}$ was deduced from Eq. (5) by using the approximation $y_{L}^{C G} \cong y_{L}-l_{S} \cdot \psi_{L}$, as is shown in Fig. 2:

$$
y_{l}=y_{L}+\left(l_{f}-l_{S}\right) \psi_{L}+\frac{a}{2}, \quad y_{r}=y_{L}+\left(l_{f}-l_{S}\right) \psi_{L}-\frac{a}{2} .
$$

From Eq. (6), the condition that the coordinates of the front wheels $y_{l}$ and $y_{r}$ are located simultaneously inside the fixed center lane strip $\pm d$ can been written as:

$$
-\frac{2 d-a}{2} \leq y_{L}+\left(l_{f}-l_{S}\right) \psi_{L} \leq \frac{2 d-a}{2}
$$

$\overline{2}$ The lateral offsets $y_{L}^{C G}$ and $y_{L}$ are considered positive on the left side of the lane. The relative yaw angle $\psi_{L}$ is considered positive for trigonometric rotations with the origin in the centerline.

3 Values for the parameters $l_{f}$ and $a$ are given in Table 2 at the end of the paper. 
Hence, the state $\mathbf{x}$ that fulfills the above inequalities (7) belongs to the set

$$
L(\overline{\mathbf{F}}) \triangleq\left\{\mathbf{x} \in \mathbb{R}^{6}:|\overline{\mathbf{F}} \mathbf{x}| \leq 1\right\}
$$

where $\overline{\mathbf{F}} \in \mathbb{R}^{1 \times 6}, \overline{\mathbf{F}}=\left(0,0, \frac{2\left(l_{f}-l_{S}\right)}{2 d-a}, \frac{2}{2 d-a}, 0,0\right)$. This set contains the state space region between two parallel hyperplanes, which are characterized by the vector $\overline{\mathbf{F}}$ and $|\overline{\mathbf{F}} \mathbf{x}|=1$, as shown in Fig. 3 .

Remark 2 As detailed later in Section 3.3, a situation is considered to be dangerous when at least one of the two front wheels crosses one of the edges of the center lane strip $\pm d$, which means $|\overline{\mathbf{F}} \mathbf{x}|=1$.

A second characteristic defining "normal driving" is that the vehicle state $\mathbf{x}$ remains in a bounded space region. Supposing that $\left|x_{i}\right| \leq x_{i}^{N}$ for $i=1, \ldots, 6$, where $x_{i}$ denotes the $i$-th component of the state vector $\mathbf{x}$, then for "normal driving" the state vector $\mathbf{x}$ belongs to the set

$$
L\left(\mathbf{F}^{N}\right) \triangleq\left\{\mathbf{x} \in \mathbb{R}^{6}:\left|\mathbf{f}_{i}^{N} \mathbf{x}\right| \leq 1, i=1, \ldots, 6\right\}
$$

where $\mathbf{F}^{N} \in \mathbb{R}^{6 \times 6}, \mathbf{f}_{i}^{N}$ represent the rows of $\mathbf{F}^{N}, f_{i, i}^{N}=\left(x_{i}^{N}\right)^{-1}$ and $f_{i, j}^{N}=0$ for $i \neq j$, $i, j=1, \ldots, 6 . L\left(\mathbf{F}^{N}\right)$ represents a hypercube in the state space characterized by the diagonal matrix $\mathbf{F}^{N}$.

Hence, the formal description of "normal driving" defined by the two above sets is $\mathbf{x} \in L(\mathbf{F})$ (see Fig. 3), where

$$
L(\mathbf{F}) \triangleq L(\overline{\mathbf{F}}) \cap L\left(\mathbf{F}^{N}\right)=\left\{\mathbf{x} \in \mathbb{R}^{6}:\left|\mathbf{f}_{i} \mathbf{x}\right| \leq 1, \mathbf{f}_{i}=\mathbf{f}_{i}^{N}, i=1, \ldots, 6, \mathbf{f}_{7}=\overline{\mathbf{F}}\right\}
$$

$L(\mathbf{F})$ represents a finite polyhedron in the state-space (a polytope) characterized by the matrix $\mathbf{F} \in \mathbb{R}^{7 \times 6}, \mathbf{F}=\left(\left(\mathbf{F}^{N}\right)^{T}, \overline{\mathbf{F}}^{T}\right)^{T}$. 


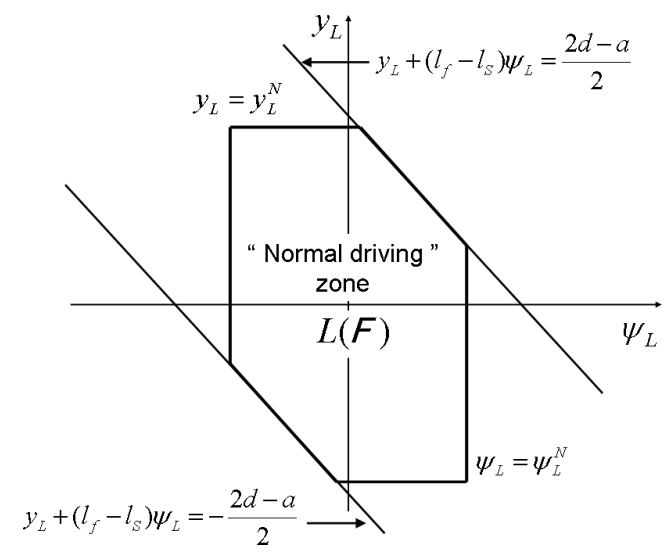

Fig. 3. "Normal driving" zone represented in a two-dimensional state space $\left(\left(y_{L}, \psi_{L}\right)\right)$.

\subsection{Switching strategy}

The steering assistance system was designed to switch on only in particular situations. This establishes two distinct continuous time systems, which represent the vehicle consecutively. One of the systems, $\Sigma_{1}$, describes the vehicle controlled by the driver alone: $\dot{\mathbf{x}}=\mathbf{A} \cdot \mathbf{x}+\mathbf{B} \cdot T_{d}$, while the other, $\Sigma_{2}$, reflects the vehicle lateral motion under the automatic steering assistance system, and perhaps influenced by the inattentive driver: $\dot{\mathbf{x}}=\mathbf{A} \cdot \mathbf{x}+\mathbf{B} \cdot\left(T_{a}+T_{d}\right)$. The transitions between $\Sigma_{1}$ and $\Sigma_{2}$ are considered instantaneous and depend on the driver's attention and on the danger for lane departure.

For the measure of the driver's attention level, the readers are referred to the concept of driver monitoring (Petersson et al. (2005)), (Bullock and Zelek (2005)). In the present paper, it is assumed that only the driver torque $T_{d}$ on the steering wheel is accessible to evaluate the driver's attention. More specifically, it is assumed that the driver is inattentive for an applied torque below a threshold $\sigma_{1},\left|T_{d}\right|<\sigma_{1}$, and is attentive otherwise. However, the analysis presented here remains valid if another variable, or threshold, is chosen to measure the driver's attention, as long as it yields the result of an "attentive" or "not attentive" driver. 
The transition from $\Sigma_{1}$ to $\Sigma_{2}$ switches on the assistance system when a driver's lack of attention during "normal driving" may lead to an unintended lane departure ${ }^{4}$ :

$$
T_{r}^{12}:\left(\left|T_{d}\right|<\sigma_{1}\right) \wedge(\mathbf{x} \in(L(\mathbf{F}))) \wedge(|\overline{\mathbf{F}} \mathbf{x}|=1) .
$$

The steering control system has to be switched off (transition from $\Sigma_{2}$ to $\Sigma_{1}$ ) whenever the driver recovers attention but, for safety reasons, should occur only if the vehicle is in the "normal driving" zone. However, to handle the case of an emergency situation, the assistance should be removed whenever the driver considers it necessary and applies a strong torque $\left|T_{d}\right| \geq \sigma_{2}$ to the steering wheel. The associated logical condition is:

$$
T_{r}^{21}:\left[\left(\sigma_{1} \leq\left|T_{d}\right|<\sigma_{2}\right) \wedge(\mathbf{x} \in(L(\mathbf{F}))] \vee\left(\left|T_{d}\right| \geq \sigma_{2}\right)\right.
$$

\section{Control law design}

This section proposes a control law for the steering torque provided by the DC motor mounted on the steering column. The main requirements for this control law are closed loop asymptotic stability, minimal overshoot with respect to the fixed center lane strip of width $2 d$, and passengers' comfort. For the simplicity of the computation and the implementation, a linear state feedback control is chosen with a compensation for the driver's torque: $T_{a}=\mathbf{K} \mathbf{x}-T_{d}$. Thus, the closed loop system $\dot{\mathbf{x}}=(\mathbf{A}+\mathbf{B K}) \mathbf{x}$ is obtained from Eq. (1). The following paragraphs express the control requirements as LMI inequalities that allow the computation of the vector

K.

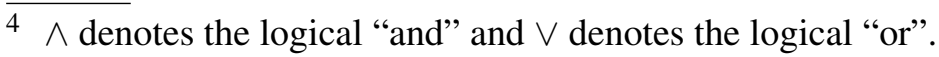




\subsection{Asymptotic stability of the closed loop system}

Concerning the system stability, the existence of a Lyapunov function $V(\mathbf{x})=\mathbf{x}^{T} \mathbf{P} \mathbf{x}$ with $\mathbf{P}$ a symmetric, positive definite matrix satisfying $(\mathbf{A}+\mathbf{B K})^{T} \mathbf{P}+\mathbf{P}(\mathbf{A}+\mathbf{B K}) \prec$ 0 guarantees the asymptotic stability of the system $\Sigma_{2}$. With the bijective transformation $\mathbf{Q}=\mathbf{P}^{-1}$ and $\mathbf{Y}=\mathbf{K} \mathbf{Q}$, the above nonlinear matrix inequality becomes linear (Boyd et al. (1994)) ${ }^{5}$ :

$$
\mathbf{Q} \mathbf{A}^{T}+\mathbf{A} \mathbf{Q}+\mathbf{B Y}+\mathbf{Y}^{T} \mathbf{B}^{T} \prec 0
$$

\subsection{Minimum overshoot with respect to the fixed center lane strip}

The ideal behavior of the minimal overshoot problem would be zero overshoot. That would be possible if the system $\Sigma_{2}$ accepted $L(\mathbf{F})$ as a polyhedral invariant set, since in this case each trajectory that starts in the set $L(\mathbf{F})$ would remain inside it (for details on the invariant set theory, see (Blanchini (1999))). As this control objective is difficult to attain for physical and control design reasons, an outer invariant approximation of the polyhedron $L(\mathbf{F})$ is used. This approximation is given by an ellipsoidal set (Minoiu et al. (2006)).

Therefore, an ellipsoid $\varepsilon=\left\{\mathbf{x}: \mathbf{x}^{T} \mathbf{P x} \leq 1\right\}$ is first sought such that it is included in $L(\mathbf{F})$ and is located as close as possible to the control activation zone $(|\overline{\mathbf{F}} \mathbf{x}|=$ 1) $\cap L\left(\mathbf{F}^{N}\right)$ (see Fig. 4). These constraints can be written as LMI expressions ( $\mathrm{Hu}$ and Lin (2000)):

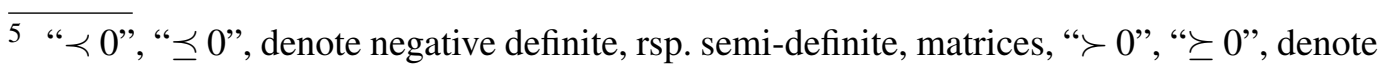
positive definite, rsp. semi-definite, matrices. 
(1) Inclusion of the ellipsoid $\varepsilon$ in $L(\mathbf{F})$.

$$
\left(\begin{array}{cc}
1 & \mathbf{f}_{i} \mathbf{Q} \\
\left(\mathbf{f}_{i} \mathbf{Q}\right)^{T} & \mathbf{Q}
\end{array}\right) \succeq 0, i=1, \ldots, 7,
$$

where $\mathbf{f}_{i}$ are the row vectors of the matrix $\mathbf{F}$ in Eq. (10).

(2) Approaching the activation zone $(|\overline{\mathbf{F}} \mathbf{x}|=1) \cap L\left(\mathbf{F}^{N}\right)$.

$$
\begin{array}{cc}
\operatorname{minimize} & -\alpha \\
\text { subject to } & \alpha \preceq \overline{\mathbf{F}}^{T} \mathbf{Q} \overline{\mathbf{F}} \\
& \overline{\mathbf{F}}^{T} \mathbf{Q} \overline{\mathbf{F}} \prec 1 .
\end{array}
$$

Once found, the ellipsoid $\varepsilon$ is expanded until it includes the above mentioned control activation zone, that is $\left((|\overline{\mathbf{F}} \mathbf{x}|=1) \cap L\left(\mathbf{F}^{N}\right)\right) \subset \varepsilon_{\text {ext }}=\left\{\mathbf{x}: \mathbf{x}^{T} \mathbf{P} x \leq V_{\text {ext }}\right\}$ (Fig. 4). This computation is achieved by maximizing the nonlinear function $V(\mathbf{x})$ under linear constraints:

$$
\begin{aligned}
& \qquad V_{\text {ext }}=\max (V(\mathbf{x}))=\max \left(\mathbf{x}^{T} \mathbf{P} \mathbf{x}\right) \\
& \text { subject to } \quad \overline{\mathbf{F}} \mathbf{x}=1 \\
& \quad \mathbf{x} \in L\left(\mathbf{F}^{N}\right) .
\end{aligned}
$$

The LMI optimization problem given in Eq. (15) provides a minimum expansion of the interior ellipsoid $\varepsilon$ to $\varepsilon_{\text {ext }}$.

The quadratic Lyapunov function $V(\mathbf{x})=\mathbf{x}^{T} \mathbf{P x}$ guarantees that the trajectories of the system $\Sigma_{2}$ will not leave the $\varepsilon_{\text {ext }}$ state space region after the assistance system is activated. Consequently, all system trajectories that start in $(|\overline{\mathbf{F}} \mathbf{x}|=1) \cap L\left(\mathbf{F}^{N}\right)$ will not leave the ellipsoidal invariant set $\varepsilon_{\text {ext }}$. 


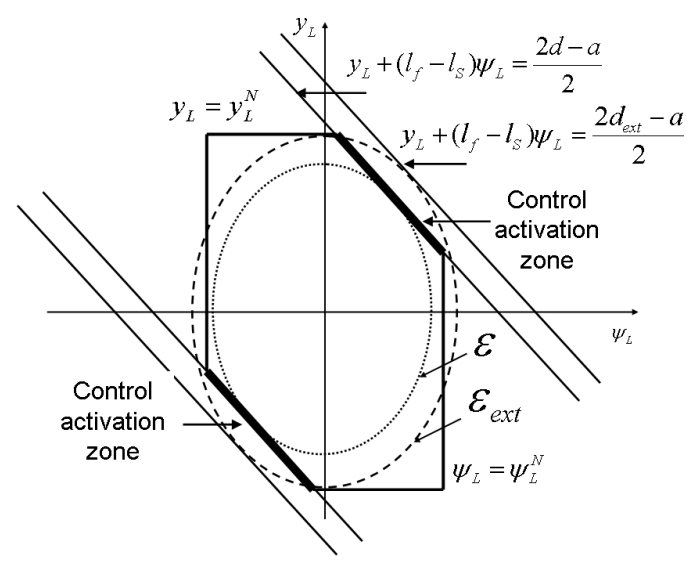

Fig. 4. The polyhedron $L(\mathbf{F})$ and the ellipsoids $\varepsilon$ and $\varepsilon_{e x t}$, represented in a two-dimensional state space $\left(y_{L}, \psi_{L}\right)$.

Furthermore, the hyperplanes tangent to $\varepsilon_{\text {ext }}$ and parallel to $|\overline{\mathbf{F}} \mathbf{x}|=1$ are equivalent to the center lane strip of width $2 d_{\text {ext }}$, where the two front wheels are guaranteed to remain during activation of the assistance system (Figs. 2, 4):

$$
d_{e x t}=\frac{2 d-a}{2} \sqrt{V_{e x t} \overline{\mathbf{F}} \mathbf{Q} \overline{\mathbf{F}}^{T}}+\frac{a}{2} .
$$

\subsection{Passengers' comfort}

For comfort reasons, and also for technical reasons, the maximum assistance torque is bounded at a pre-defined value for vehicle states $\mathbf{x} \in \varepsilon$, denoted here by $T_{M}$. This is achieved by imposing the ellipsoidal invariant set $\varepsilon$ to be contained in the polyhedron $L(\mathbf{K})=\left\{\mathbf{x} \in \mathbf{R}^{6}:|\mathbf{K} \mathbf{x}| \leq T_{M}\right\}$, which is equivalent to the following LMI condition:

$$
\left(\begin{array}{cc}
1 & \frac{1}{T_{M}} \mathbf{Y} \\
\frac{1}{T_{M}} \mathbf{Y}^{T} & \mathbf{Q}
\end{array}\right) \succeq 0 .
$$

Furthermore, by expanding the ellipsoid $\varepsilon$ to $\varepsilon_{\text {ext }}$, the guaranteed maximum torque for $\mathbf{x} \in \varepsilon_{\text {ext }}$ is greater than $T_{M}$. Therefore, the torque limit $T_{M}$ was chosen to be smaller than the maximum torque supported by the DC motor. In addition, an upper 
bound of the motor torque used to bring the vehicle to the correct trajectory from a vehicle state $\mathbf{x}$ in $\varepsilon_{\text {ext }}$ is given by $T_{M_{\text {ext }}}=\max _{\mathbf{x} \in \varepsilon_{\text {ext }}}(\mathbf{K} \mathbf{x})=\sqrt{V_{\text {ext }}} \sqrt{\mathbf{K} \mathbf{P}^{-1} \mathbf{K}^{T}}$.

Putting together the conditions from the above paragraphs 4.1, 4.2, and 4.3, the feedback control vector $\mathbf{K}$ based on the Lyapunov function $V(\mathbf{x})=\mathbf{x}^{T} \mathbf{P x}$ can be obtained as a result of the following LMI linear cost optimization problem.

$$
\begin{array}{ll}
\text { minimize } & -\alpha \\
\text { subject to } & E q .(13) \quad \text { (system stability), } \\
& E q .(14) \quad \text { (inclusion in the "normal driving" set), } \\
& E q .(15) \quad \text { (approaching the activation zones), } \\
& E q .(18) \quad \text { (bounding the steering torque). }
\end{array}
$$

This LMI optimization problem has $\mathbf{Q}$ and $\mathbf{Y}$ as matrix variables. $\mathbf{P}=\mathbf{Q}^{-1}$ and $\mathbf{K}=\mathbf{Q}^{-1} \mathbf{Y}$ are obtained afterwards.

The range of the state variables during the assistance intervention can also be considered to reflect the passengers' comfort. This range can be computed only a posteriori, after the controller design is completed. Therefore, multiple iterations may potentially be required to achieve acceptable values. Upper bounds for these variables are given by projecting the ellipsoid $\varepsilon_{\text {ext }}$ on the six state coordinates. These projections are defined by $x_{i}^{M}$, where $\left|x_{i}\right| \leq x_{i}^{M}, x_{i}^{M}=\sqrt{Q_{i, i}}$ for $i=1, \ldots, 6$ and $Q_{i, i}$ is an element of the diagonal of matrix $\mathbf{Q}$. 


\subsection{Robustness against vehicle speed and adhesion variations}

The system description given in Eq. (1) depends nonlinearly on the vehicle speed $v$. The control law developed in Section 4 provides the required performance only for a fixed predefined longitudinal velocity $v$. In this part of the paper, a valid extension of the control law to a speed interval is proposed.

By choosing $\xi_{v} \in[-1 ; 1]$, a parameter that describes the variation of $v$ between a lower limit $v_{\min }$ and an upper limit $v_{\max }$, the following can be written (Raharijoana (2004)):

$$
\begin{aligned}
& \frac{1}{v}=\frac{1}{v_{0}}+\frac{1}{v_{1}} \xi_{v}, \quad v \cong v_{0}\left(1-\frac{v_{0}}{v_{1}} \xi_{v}\right), \\
& \frac{1}{v^{2}} \cong \frac{1}{v_{0}^{2}}\left(1+2 \frac{v_{0}}{v_{1}} \xi_{v}\right) .
\end{aligned}
$$

Setting $\xi_{v}=-1 v=v_{\min }$ and $\xi_{v}=1 v=v_{\max }, v_{0}$ and $v_{1}$ can be written as following:

$$
v_{0}=\frac{2 v_{\min } v_{\max }}{v_{\max }+v_{\min }}, v_{1}=-\frac{2\left(v_{\min } v_{\max }\right)}{v_{\max }-v_{\min }} .
$$

With these expressions for $v, 1 / v$, and $1 / v^{2}$, the matrix $\mathbf{A}$ of the system given in Eq. (1) can be written as $\mathbf{A}=\mathbf{A}^{*}+\mathbf{A}^{* *} \xi_{v}$. Hence, the matrix $\mathbf{A}(v)$ evolves into a matrix polytope for $v \in\left[v_{\min }, v_{\max }\right]$.

The LMI optimization problem given in Eq. (19) of Section 4 can be modified for a varying vehicle speed $v \in\left[v_{\min }, v_{\max }\right]$. Indeed, only the matrix $\mathbf{A}$ of the LMI problem is speed dependent. Due to the linear convex property of the matrix polytope, ineq. (13) holds for any $v \in\left[v_{\min }, v_{\max }\right]$ if the following holds:

$$
\mathbf{Q}\left(\mathbf{A}^{*} \pm \mathbf{A}^{* *}\right)^{T}+\left(\mathbf{A}^{*} \pm \mathbf{A}^{* *}\right) \mathbf{Q}+\mathbf{B Y}+\mathbf{Y}^{T} \mathbf{B}^{T} \prec 0 .
$$

The above considerations mean that if the matrix variables $\mathbf{Q}$ and $\mathbf{Y}$ are obtained such that they minimize the LMI problem from Eq. (19), for both $\mathbf{A}=\mathbf{A}^{*}-\mathbf{A}^{* *}$ and 
for $\mathbf{A}=\mathbf{A}^{*}+\mathbf{A}^{* *}$, then the control vector $\mathbf{K}$ stabilizes the system given in Eq. (1) for any varying speed $v \in\left[v_{\min }, v_{\max }\right]$, while satisfying the required performance.

As condition (22) is conservative, it may lead to poor performance over the speed range. Thus, taking into account many speed intervals $\left[v_{\text {min }}^{i}, v_{\text {max }}^{i}\right],\left[v_{\text {min }}^{i+1}, v_{\text {max }}^{i+1}\right], v_{\text {min }}^{i+1}<$ $v_{\text {max }}^{i}$, and the corresponding feedback vectors $\mathbf{K}^{i}, \mathbf{K}^{i+1}$, gain scheduling can be carried out (Stilwell and Rugh (1997)).

The same reasoning can be used for the adhesion $\mu$ as for the vehicle speed. Furthermore, the matrix $\mathbf{A}$ already depends linearly on $\mu$. Considering that $\mu \in$ $\left[\mu_{\min }, 1\right]$, the vertices of the new matrix polytope are obtained for $\left\{\mu_{\min }, 1\right\}$. If variations of both $v$ and $\mu$ are considered, the matrix $\mathbf{A}$ becomes multi-affine, and thus a Linear Fractional Representation (LFR) can be adopted (Scherer et al. (1997)).

\section{Discussion of the trajectories of the switched system driver-steering assis- tance}

The trajectories of the switched system are briefly analyzed in this Section. This explains the choice of the switching strategy in Section 3.3 and of the control law in Section 4.

By design, the set $\varepsilon_{\text {ext }}$ contains the control activation zones $(|\overline{\mathbf{F}} \mathbf{x}|=1) \cap L\left(\mathbf{F}^{N}\right)$. However, the steering assistance system switches on if one of these zones is crossed, and hence is inside $\varepsilon_{e x t}$. If the driver recovers attention, in which case $\sigma_{1} \leq\left|T_{d}\right|<$ $\sigma_{2}$, or if the driver requires an urgent deactivation $\left(\left|T_{d}\right| \geq \sigma_{2}\right)$, the switching off of the steering assistance system always takes place inside $\varepsilon_{\text {ext }}$ (due to the invariant set property of $\left.\varepsilon_{e x t}\right)$. 
The driver then regains control of the vehicle. Excursions outside the invariant set $\varepsilon_{e x t}$ are very likely to occur when the driver undertakes maneuvers outside of "normal driving". The assistance activation will in this case always be inhibited, independent of the driver's attention, according to the switching rules. If the driver performs maneuvers outside "normal driving" (for instance, lane changing or cornering), during the subsequent lane following the vehicle's trajectory will evolve into the "normal driving" set $L(F)$. The next assistance activation will occur necessarily within the activation zone and the reasoning can be repeated. Thus, it can be concluded that the switching does not induce the divergence of the vehicle trajectory.

\section{Driving test results}

\subsection{Control law computation}

For the control law computation, bounds defining "normal driving" were fixed. In the literature, few statistical studies about the variation ranges of vehicle's state variables for a lane keeping task exist (Pilutti and Ulsoy (1999)), (Pomerleau et al. (1999)). The bounds used in this paper take into account values given by (Bar and Page (2002)), who analyzed accidents due to different types of lane departure. The limits defining the set $L\left(\mathbf{F}^{N}\right)$ are given in the column $x_{i}^{N}$ of Table 1 . The center lane strip related to "normal driving" was fixed at $d= \pm 1.1 \mathrm{~m}$ with respect to the center of the lane. During testing, these values were found to be wide enough to allow to the driver safe displacement of the front wheels of the vehicle with respect to the centerline while staying in the lane. The vehicle speed was considered to vary within the interval $v \in[18 m / s ; 22 m / s]$. 
Using these values, the matrix $\mathbf{P}$ and the vector $\mathbf{K}$ were computed. The resulting vector $\mathbf{K}$ is given by:

$$
\mathbf{K}=(-198.5,-69.3,-355.9,-17.7,-409.9,5.5) .
$$

The closed loop vehicle model was simulated for $v \in[18 \mathrm{~m} / \mathrm{s} ; 22 \mathrm{~m} / \mathrm{s}]$ and the system poles were computed. The system has two real poles and two pairs of complex conjugate poles. All the poles have their real parts located to the left of -0.6 in the complex plane. The damping factors remain below 1.18, showing good robustness properties.

The system trajectories are guaranteed to remain below the limits given in the column $x_{i}^{M}$ of Table 1 during activation of the control system. The upper bound for the assistance steering torque was found to be $26.22 \mathrm{Nm}$. According to the numerical results, the trajectories of the front wheels of the vehicle do not exceed $d_{\text {ext }}=1.76 \mathrm{~m}$ during the assistance system activation. It was assumed that the driver is inattentive for a steering torque $\sigma_{1}$ below $2 \mathrm{Nm}$, and the emergency deactivation limit $\sigma_{2}$ was set to $6 \mathrm{Nm}$.

\subsection{Test environment}

Tests were conducted on a track located in Satory, $20 \mathrm{~km}$ west of Paris, France. The track is $3.5 \mathrm{~km}$ long with various road profiles including a straight lane and tight bends. The experimental vehicle was equipped with a CORREVIT sensor that measures the side slip angle $\beta$, an Inertial Navigation System to measure the yaw rate $r$, and an odometer for the vehicle longitudinal velocity $v$. The steering angle $\delta_{f}$ was obtained from an optical encoder and its derivative $\dot{\delta}_{f}$ was computed numerically. The driver torque was measured by a load cell sensor integrated into the steering wheel. The assistance torque was provided by a DC motor mounted 
Table 1

Bounds $x_{i}^{N}$ corresponding to the "normal driving" set and guaranteed bounds by control design $x_{i}^{M}$ and $\left(x_{i}^{M}\right)_{\text {new }}$ for the cases of use of the first and second switching strategy, respectively.

\begin{tabular}{ccccc} 
& $x_{i}^{N}$ & $x_{i}^{M}$ & $\left(x_{i}^{M}\right)_{\text {new }}$ & \\
\hline$\beta$ & 0.0104 & 0.0297 & 0.0507 & $\mathrm{rad}$ \\
$r$ & 0.1047 & 0.2789 & 0.4752 & $\mathrm{rad} / \mathrm{s}$ \\
& & & & \\
$\psi_{L}$ & 0.0349 & 0.0801 & 0.1365 & $\mathrm{rad}$ \\
$y_{L}$ & 0.8 & 1.02 & 1.73 & $\mathrm{~m}$ \\
& & & & \\
$\delta_{f}$ & 0.0261 & 0.0584 & 0.0995 & $\mathrm{rad}$ \\
$\dot{\delta}_{f}$ & 0.2094 & 0.5739 & 0.9779 & $\mathrm{rad} / \mathrm{s}$ \\
\hline
\end{tabular}

on the steering column (Fig. 5(b)). The look-ahead lateral offset and the relative yaw angle were measured using a video camera that detects the lane markers using vision algorithms (Labayrade et al. (2006)) (Fig. 5(a)).

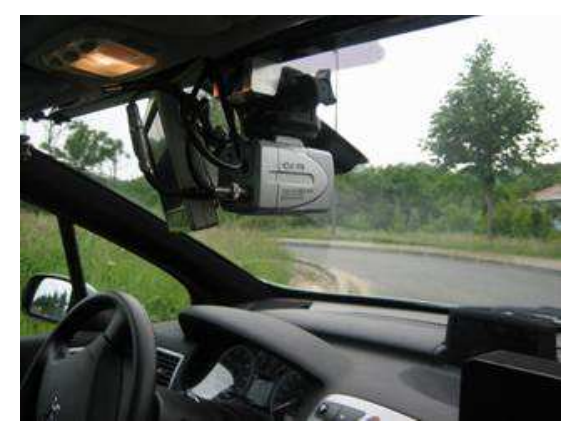

(a)

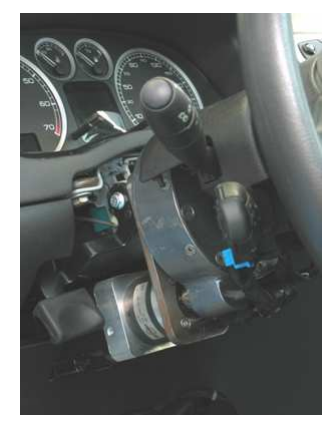

(b)

Fig. 5. (a) Video camera in the front of the vehicle. (b) DC motor on the steering column. 


\subsection{Practical implementation results}

To perform the tests, a segment of the test track with a road curvature less than $0.001 \mathrm{~m}^{-1}$, which corresponds to a nearly straight road, was chosen. The driver drove the instrumented vehicle following the center of the lane, occasionally taking his hands off the steering wheel, or relaxing the steering control to simulate a lack of attention (see Electronic Annex 1 in the online version of this article). The activation of the steering assistance was linked to a "beep" sound. Once the assistance had been activated, the driver acted on the steering wheel after a few seconds to recover the control of the vehicle.

Fig. 6 (a) shows the trajectories of the front wheels on the lane, as well as the limits for the control activation at $\pm 1.1 \mathrm{~m}$, the guaranteed overshoots limits at $\pm 1.76 \mathrm{~m}$, and the border of the lane at $\pm 1.75 m$ with respect to the center of the lane ${ }^{6}$. It is apparent that after activation of the control system, the trajectories of the front wheels did not cross the border of the lane. Moreover, they remained within the normal driving zone, inside the $\pm 1.1 \mathrm{~m}$ lane strip. At the first reaction after the system activation, the assistance system counter-steered using a torque of about $10 \mathrm{Nm}$ (see Fig. 6 (b)), and the trajectories of the front wheels occasionally crossed the opposite line of the $\pm 1.1 \mathrm{~m}$ center strip. Subsequently, the vehicle was driven to the center of the lane and experienced low frequency, small amplitude oscillations around the centerline. The vehicle speed during the test varied between $18 \mathrm{~m} / \mathrm{s}$ and

\footnotetext{
$\overline{6}$ In each figure that presents the test results, the assistance activation is represented by a two-valued figure; the higher value corresponds to the situation in which the driver controls the vehicle, while the lower value corresponds to the situation in which the assistance system controls the vehicle. For better understanding, the two values are different for each figure and are adapted to the figure scale.
} 
$22 \mathrm{~m} / \mathrm{s}$.

Note that the assist torque represented in Fig. 6(b) is higher than the driver torque, since in manual mode the driver is assisted by the motor itself, which works in this mode as a classical electrically powered assistance (Electric Power Steering, EPS). The driver torque is measured at the steering wheel, which is upstream of the EPS multiplication factor. In Fig. 6(b) the driver's torque is represented, and is not the result of the driver's torque multiplied by the EPS.

During the intervention of the assistance system, the electric motor provides the necessary steering torque, which results from the control law. It was assumed that during the assistance system intervention, the driver is not able to drive. The assistance system takes over for the driver, but it is switched off as soon as the driver recovers attention. Consequently, the driver feels the assistance acting on the steering wheel only during the moments of system deactivation. At these times, the driver feels a small resistance in the steering wheel, which disappears quickly.

These results confirmed the expected performance, which is guaranteed by the control design method. In addition, it was noticed that the computed maximum bounds are fairly conservative compared to the real data. This might be due to an overestimation of the polyhedron $L(F)$ by the exterior ellipsoid $\varepsilon_{\text {ext }}$. Consequently, an important shortcoming of the above presented assistance system, the activation conditions, could be improved. By intensive testing, it was noticed that the activation rules are sometimes too conservative and prevent the steering assistance from switching on, as for example in Fig. 6 (a), at $t=112 s$. Therefore, section 7 presents an alternative switching strategy. 


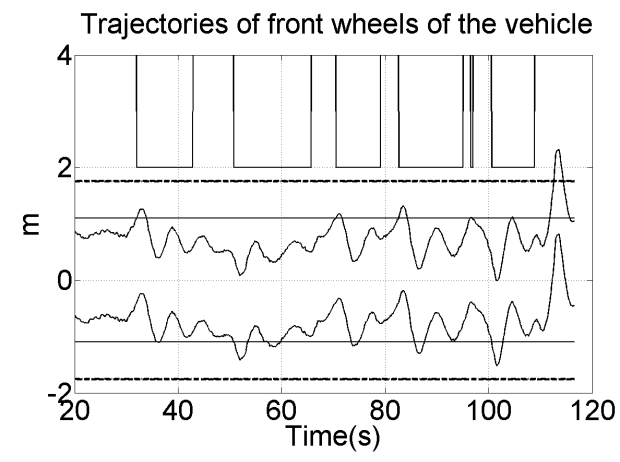

(a)

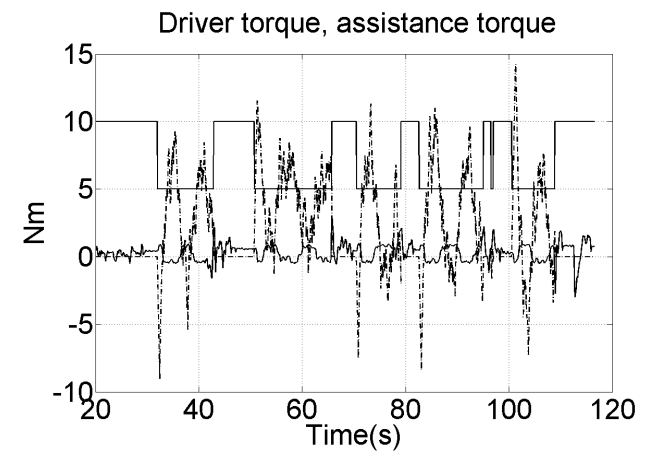

(b)

Fig. 6. (a) Trajectories of the front wheels (solid line), predefined lane strip $\pm d= \pm 1.1 \mathrm{~m}$ (solid line), computed driving lane strip $\pm d_{\text {ext }}= \pm 1.76 \mathrm{~m}$ (dashed line), lane borders at $\pm 1.75 \mathrm{~m}$ (dash-dot line, this coincides with the previous dashed line), assistance activated on 2 (first activation rules). (b) Driver's torque (solid line), assistance torque (dash-dot line), assistance activated on 5 (first activation rules).

\section{Improving the switching strategy and new testing}

\subsection{New activation rules}

In examining the activation/deactivation situations during the tests, it was noticed that when slowly drifting out of the lane with a relatively small yaw angle and yaw rate, the condition that the vehicle state still resides in the "normal driving" zone is always satisfied when the vehicle leaves the $\pm d$ center lane strip. On the contrary, for rapid lane departures, the steering assistance did not switch on because the "normal driving" zone had previously been exceeded by the vehicle.

Hence, the developed control law was kept unchanged and the constraint $\mathbf{x} \in L\left(\mathbf{F}^{N}\right)$ was eliminated from the switching condition $T_{r}^{12}$ given in Eq. (11), while the constraint $|\overline{\mathbf{F}} \mathbf{x}|=1$ (crossing of the $\pm d$ center lane strip) was maintained. Nevertheless, to satisfy some safety limits for the lateral displacement of the front wheels, a new 
activation condition was added. It required that for any activation, the maximum expected displacements of the front wheels $\tilde{d}_{e x t}(\mathbf{x})$ would be less than $\tilde{d}_{e x t}=2.5 \mathrm{~m}$. Taking into account the conservatism noticed in the experimental phase of the first switching strategy, this value was chosen to be higher than the value corresponding to the lane borders $(1.75 m)$. More specifically, for each state $\mathbf{x}$ for which the front wheels reach the lane strip limits at $\pm d$, the expected displacement $\tilde{d}_{\text {ext }}(\mathbf{x})$ can be computed by using Eq. (17) for $V_{\text {ext }}(\mathbf{x})=\mathbf{x}^{T} \mathbf{P x}$, as shown in Fig. 7. Moreover, the guaranteed maximum bounds for the state variables during the control activation were computed for the new ellipsoid $\tilde{\varepsilon}_{\text {ext }}$ related to $\tilde{d}_{\text {ext }}=2.5 \mathrm{~m}$, and are given in Table 1, column $\left(\mathbf{x}_{i}^{M}\right)_{\text {new }}$.

In addition, the transition $T_{r}^{12}$ has to be triggered only if the vehicle is heading towards a lane edge. This behavior might be characterized by a lateral offset and a yaw angle that are simultaneously positive or negative.

For the deactivation of the steering assistance, the same conditions were used as in the first switching strategy, in order to ensure safety when switching the system off and the trajectory limits with respect to the switchings.

The proposed new switching strategy is illustrated in Fig. 7 and summarized in the following logical equations:

$$
\begin{gathered}
\left.T_{r}^{12}:\left(\left|T_{d}\right|<\sigma_{1}\right) \wedge\left(\tilde{d}_{e x t}(\mathbf{x})<2.5 m\right) \wedge(|\overline{\mathbf{F}} \mathbf{x}|=1) \wedge\left(\psi_{L} \cdot y_{L}\right)>0\right) \\
T_{r}^{21}:\left[\left(\sigma_{1} \leq\left|T_{d}\right|<\sigma_{2}\right) \wedge(\mathbf{x} \in(L(\mathbf{F}))] \vee\left(\left|T_{d}\right| \geq \sigma_{2}\right) .\right.
\end{gathered}
$$




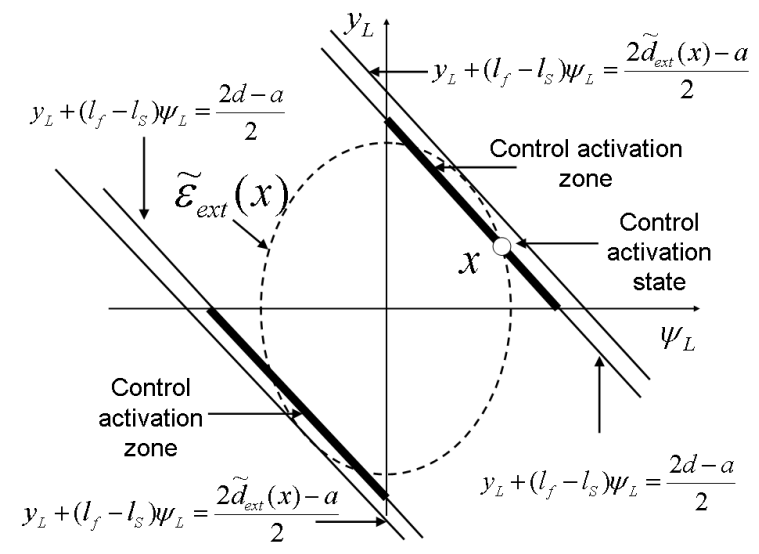

Fig. 7. Control activation for the new switching strategy.

\subsection{Practical implementation}

During the testing of the second switching strategy, the driver followed the lane and occasionally applied a strong torque on the steering wheel in an attempt to force drifting from the lane. He then removed his hands from the steering wheel. Under these conditions, the state variables were forced to approach and exceed the "normal driving" zone at the crossing of the $\pm d$ lane strip bounds. With this new strategy, the activation of the steering assistance system occurred for almost all fast lane departures, except for very severe cases. In general, the expected maximum lateral offset of the front wheels, $\tilde{d}_{\text {ext }}(\mathbf{x})$, stayed below $2.5 \mathrm{~m}$. Fig. 8 (a) shows that the front wheels of the vehicle exceeded the lane borders, but they remained below $2 m$ with respect to the center of the lane. The expected theoretical value of $\tilde{d}_{\text {ext }}(\mathbf{x})$ again turned out to be conservative with respect to the experimental results. Figs. 9 to 11 show that the state variables remained below their maximum expected values given in Table 1, column $\left(x_{i}^{M}\right)_{\text {new }}$. The limits of the "normal driving" zone are also represented as dashed lines in these Figs. 9 to 11 in order to get show the difference between the guaranteed maximum bounds and the recorded data. 


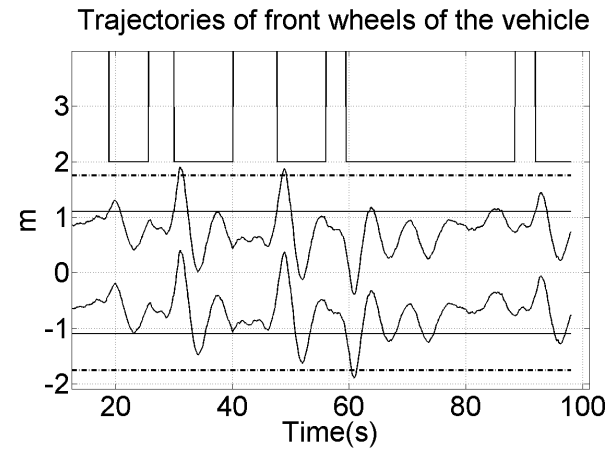

(a)

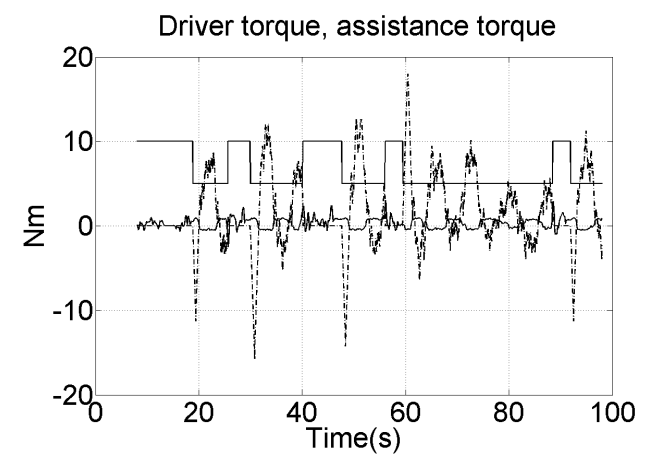

(b)

Fig. 8. (a) Trajectories of the front wheels (solid line), predefined lane strip $\pm d= \pm 1.1 \mathrm{~m}$ (solid line), lane border (dash-dot line), assistance activated on 2 (second activation rules). (b) Driver torque (solid line), assistance torque (dash-dot line), assistance activated on 5 (second activation rules).

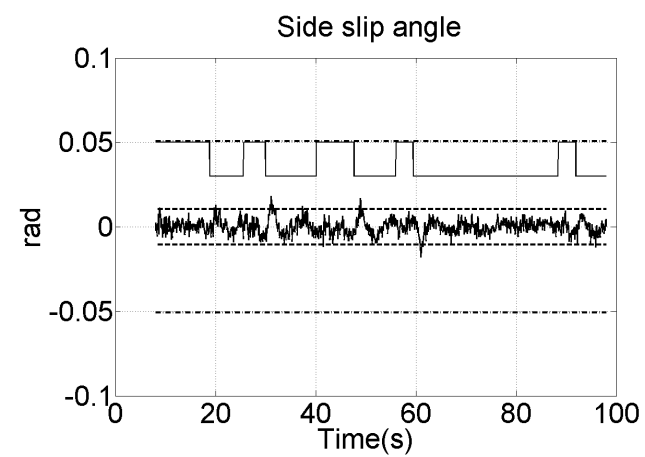

(a)

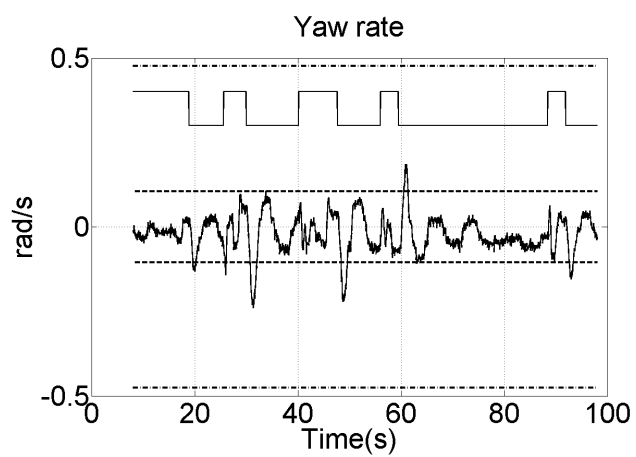

(b)

Fig. 9. (a) Side slip angle $\beta$ (solid line), "normal driving" value $\beta^{N}$ (dashed line), maximum computed bound $\beta^{M}$ (dash-dot line), assistance activated on 0.03 (second activation rules). (b) Yaw rate $r$ (solid line), "normal driving" value $r^{N}$ (dashed line), maximum computed bound $r^{M}$ (dash-dot line), assistance activated on 0.3 (second activation rules).

\subsection{Comparison of the activation rules}

In order to compare the activation of the two switching strategies, the following test was performed. In the first stage, the equipped vehicle was driven on the test track 


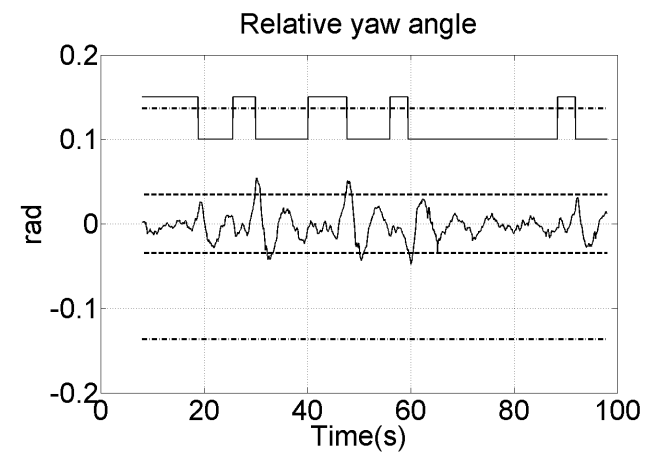

(a)

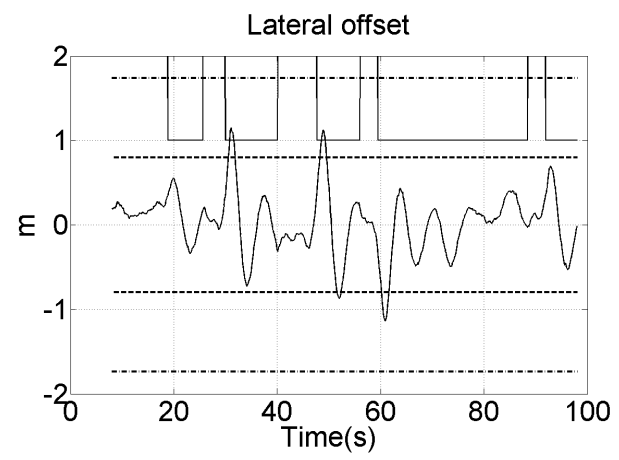

(b)

Fig. 10. (a) Relative yaw angle $\psi_{L}$ (solid line), "normal driving" value $\psi_{L}^{N}$ (dashed line), maximum computed bound $\psi_{L}^{M}$ (dash-dot line), assistance activated on 0.1 (second activation rules). (b) Lateral offset $y_{L}$ (solid line), "normal driving" value $y_{L}^{N}$ (dashed line), maximum computed bound $y_{L}^{M}$ (dash-dot line), assistance activated on 1 (second activation rules).

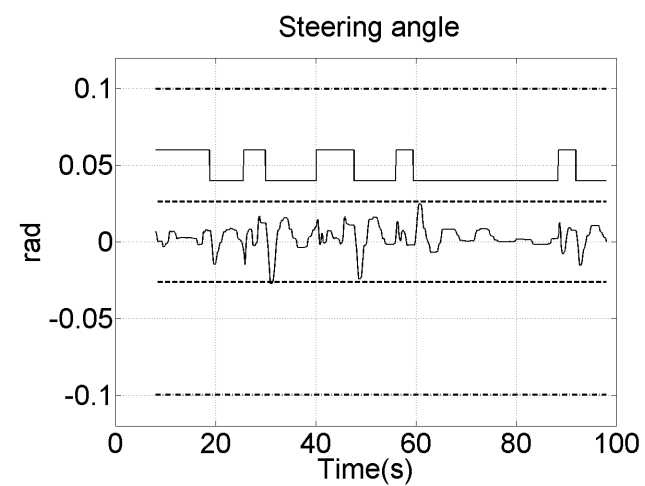

(a)

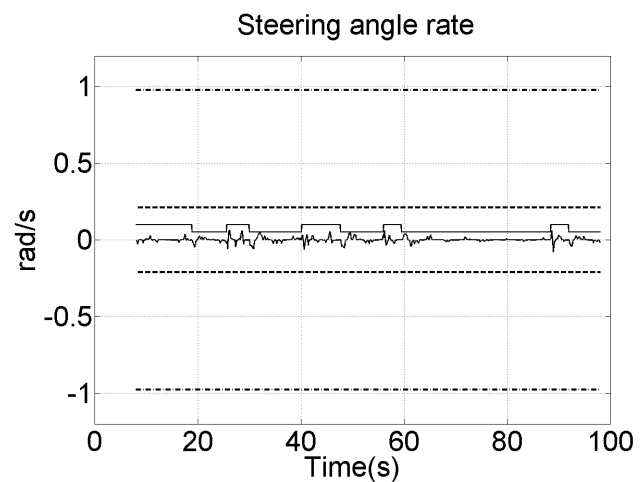

(b)

Fig. 11. (a) Steering angle $\delta_{f}$ (solid line), "normal driving" value $\delta_{f}^{N}$ (dashed line), maximum computed bound $\delta_{f}^{M}$ (dash-dot line), assistance activated on 0.04 (second activation rules). (b) Steering angle rate $\dot{\delta}_{f}$ (solid line), "normal driving" value $\dot{\delta}_{f}^{N}$ (dashed line), maximum computed bound $\dot{\delta}_{f}^{M}$ (dash-dot line), assistance activated on 0.05 (second activation rules). 
with the steering assistance system disabled, and it was allowed to leave the lane, with the driver simulating a lack of attention. Subsequently, the vehicle trajectory was corrected by the driver to the center of the lane, and the procedure was repeated several times. All the variables necessary for the computation of the switching conditions were recorded during the test. In the second stage, the activation conditions were computed off-line for the two switching strategies, and were compared to each other. The deactivation condition was reduced to a driver torque higher than $1.5 \mathrm{Nm}$, to enforce a rapid deactivation of the system. The trajectories of the front wheels are given in Fig. 12 (a). There are three cases of activation using the second strategy in the absence of system activation using the first strategy. In all three cases, the absence of the activation for the first switching strategy is due to a yaw rate that exceeded its normal value $r^{N}$ (see Fig. 12 (b)). This result raises several questions concerning the driving behavior during a "normal driving" situation and the moment of loss of attention. As few statistical studies providing bounds of the vehicle state variables during a usual lane keeping task are available, it is thus difficult to determine whether these missed activations for the first switching strategy are dangerous or not.

To summarize, it can be noticed that with the two activation strategies of the steering assistance system, there is a place for calibrations and settings that take users' opinions into account. The first activation conditions are more restrictive, but they ensure a vehicle trajectory that is confined to the lane while remaining within comfortable and safe limits for the state variables during the control activation. The second activation rules are more reactive and cover fast drifting from the lane, but the danger to depart from the lane to collide with adjacent vehicles cannot be excluded. 


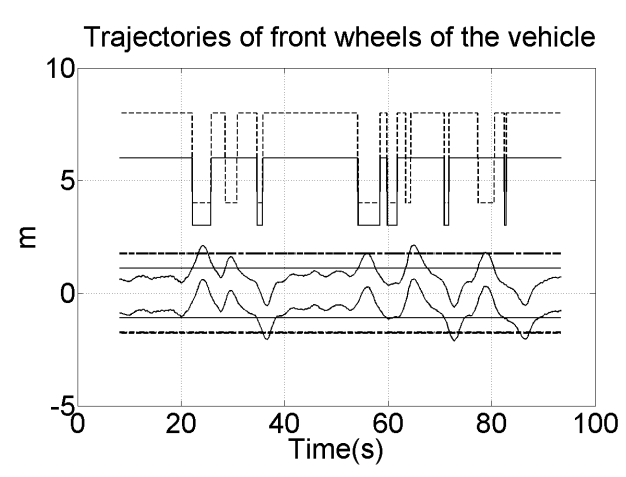

(a)

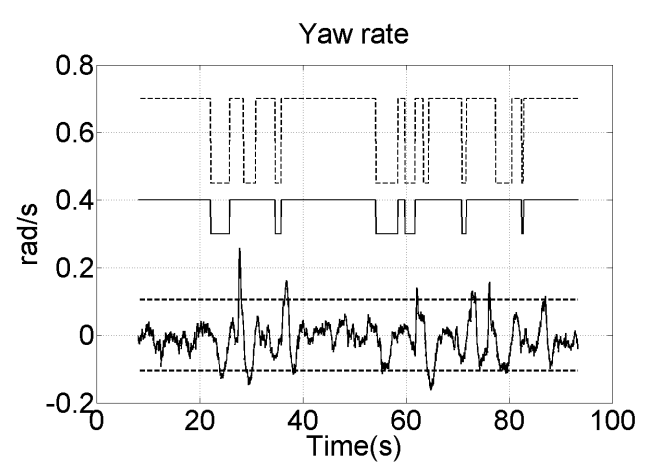

(b)

Fig. 12. (a) Trajectories of the front wheels (solid line), predefined lane strip $\pm d= \pm 1.1 \mathrm{~m}$ (solid line), lane border (dash-dot line), assistance activated for the first switching strategy on 3 (solid line), assistance activated for the second switching strategy on 4 (dashed line). (b) Yaw rate $r$ (solid line), "normal driving" value $r^{N}$ (dashed line), assistance activated for the first switching strategy on 0.3 (solid line), assistance activated for the second switching strategy on 0.45 (dashed line).

\section{Conclusions and future work}

This paper presented the design and implementation of an automatic lane keeping assistance system in a prototype passenger vehicle. The steering assistance system switches on as soon as the front wheels cross a center lane strip, and brings the vehicle back to the center of the lane.

The lateral control law is based on a new concept linking Lyapunov theory with LMI optimization. It ensures four important features during its intervention: asymptotic convergence of the vehicle trajectory to the lane centerline, a guarantee that the front of the vehicle remains inside a safety zone within the lane during the assistance activation, bounded vehicle dynamics, and control torque input.

For the switching strategy, two activation rules with different degrees of reaction with respect to the driver's actions and vehicle position have been proposed, im- 
plemented on the prototype vehicle, and tested. Using both rules, unintended lane departures are avoided, without leaving the lane in the first case and with a small overshoot with respect to the lane borders in the second case. As a trade-off, the second activation rule ensures better reactions for fast drifting out the lane.

In the future, we intend to perform an acceptance study of the proposed steering assistance systems using test drivers. This study could be extended by a complementary statistical study concerning the limits of the vehicle dynamic variables during "normal driving", especially if the driver experiences moments of inattention. The topic of avoiding unintended lane departure for curvy roads is a topic of current research.

\section{References}

Ackermann, J., Guldner, J., Sienel, W., Steinhauser, R. \& Utkin, V. U. (1995), Linear and Nonlinear Controller Design for Robust Automatic Steering, IEEE Trans. Control Systems Technology, 132-143.

Bar, F. \& Page, Y. (2002), Les sorties de voie involontaires, CEESAR - LAB Tech. Rep. ARCOS.

Blanchini, F. (1999), Set invariance in control, Automatica, 35, 1747-1767.

Boyd, S., El Ghaoui, L., Feron, E. \& Balakrishnan, V. (1994), Linear Matrix Inequalities in System and Control Theory, Society for Industrial and Applied Mathematics, Philadelphia.

Bullock, D. \& Zelek, J. (2005), Towards real-time 3-D monocular visual tracking of human limbs in unconstrained environments, Real-Time Imaging, 11, 323-353.

Eidehall, A., Pohl, J. \& Gustafsson, F. (2007), Joint road geometry estimation and vehicle tracking, Control Engineering Practice, 15, 1484-1494. 
Table 2

Values of the vehicle parameters.

\begin{tabular}{|c|c|c|}
\hline Parameter & & Value \\
\hline$B_{S}$ & steering system damping coefficient & 15 \\
\hline$c_{f 0}$ & front cornering stiffness & $40000 \mathrm{~N} / \mathrm{rad}$ \\
\hline$c_{r 0}$ & rear cornering stiffness & $35000 \mathrm{~N} / \mathrm{rad}$ \\
\hline$I_{S}$ & inertial moment of steering system & $0.05 \mathrm{~kg} \cdot \mathrm{m}^{2}$ \\
\hline$J$ & vehicle yaw moment of inertia & $2454 \mathrm{~kg} \cdot \mathrm{m}^{2}$ \\
\hline$K_{P}$ & manual steering column coefficient & 1 \\
\hline$l_{f}$ & distance form CG to front axle & $1.22 \mathrm{~m}$ \\
\hline$l_{r}$ & distance from CG to rear axle & $1.44 \mathrm{~m}$ \\
\hline$l_{S}$ & look-ahead distance & $0.95 \mathrm{~m}$ \\
\hline$a$ & vehicle width & $1.5 \mathrm{~m}$ \\
\hline$m$ & total mass & $1600 \mathrm{~kg}$ \\
\hline$R_{S}$ & steering gear ratio & 14 \\
\hline$v$ & longitudinal velocity & {$[18 ; 22] \mathrm{m} / \mathrm{s}$} \\
\hline$\eta_{t}$ & tire length contact & $0.13 \mathrm{~m}$ \\
\hline$\mu$ & adhesion & 1 \\
\hline$L$ & lane width & $3.5 \mathrm{~m}$ \\
\hline
\end{tabular}


Hu, T. \& Lin, Z. (2000), On enlarging the basin of attraction for linear systems under saturated linear feedback, Systems and Control Letters, 40, 59-69.

Isermann, R. (2008), Mechatronic systems - Innovative products with embedded control, Control Engineering Practice, 16, 14-29.

Labayrade, R., Douret, J., Laneurit, J. \& Chapuis, R. (2006), A reliable and robust lane detection system based on the parallel use of three algorithms for driving safety assistance, IEICE Trans. Information and Systems Online, E89-D, 20922100.

LeBlanc, D.J., Venhovens, P.J.Th., Lin, C.-F., Pilutti, T.E., Ervin, R.D., Ulsoy, A.G., MacAdam, C. \& Johnson, G.E. (1996), Warning and intervention system to prevent road-departure accidents, Vehicle System Dynamics, 25 (Suppl.), 383-396.

Minoiu, N., Netto, M. \& Mammar, S. (2006), A switched optimized approach for road departure avoidance, Proc. IEEE Conf. on Intelligent Transportation Systems, 133-138.

Minoiu, N., Netto, M., Mammar, S. \& Lusetti, B. (2007), A switched optimized approach for road-departure avoidance: implementation results, Proc. IEEE Intelligent Vehicles Symposium, 787-792.

Nagai, M., Mouri, H. \& Raksincharoensak, P. (2002), Vehicle Lane-Tracking Control with Steering Torque Input, Vehicle System Dynamics Supplement, 37, 267 278.

National Highway Traffic Safety Administration (2006), Traffic safety facts 2005, U.S. Department of Transportation, Washington.

Petersson, L., Fletcher, L. \& Zelinsky, A. (2005), A framework for driver-in-theloop driver assistance systems, Proc. IEEE Conf. Intelligent Transportation Systems, 771-776.

Pilutti, T. \& Ulsoy, A. G. (1999), Identification of driver state for lane keeping tasks, IEEE Transactions on Systems, Man, and Cybernetics-Part A: Systems 
and Humans, Vol. 29, No. 5, 486-502.

Pomerleau, D., Jochem, T., Thorpe, C., Batavia, P., Pape, D., Hadden, J., McMillan, N., Brown, N. \& Everson, J. (1999), Run-of-road collision avoidance using IVHS countermeasures, U.S. Department of Transportation National Highway Traffic Safety Administration, Technical Report DOT HS 809170.

Rossetter, E. J., Switkes, J. P. \& Gerdes, J. C. (2004), Experimental validation of the potential field lanekeeping system, Int. Journal of Automotive Technology, $5,95-108$.

Raharijoana, T. (2004), Commande robuste pour l'assistance au contrôle latéral d'un véhicule routier, $\mathrm{PhD}$ thesis, Univérsité Paris XI Orsay.

Shimakage, M., Satoh, S., Uenuma, K. \& H. Mouri (2002), Design of lane-keeping control with steering torque input, JSAE Review, 23, 317-323.

Stilwell, D.J. \& Rugh, W. J. (1997), Interpolation of Observer State Feedback Controllers for Gain Scheduling, IEEE Transaction on Automatic Control, 44, 1225 1229.

Scherer, C., Gahinet, P. \& Chilali, M. (1997), Multiobjective output-feedback control via LMI optimization, IEEE Transaction on Automatic Control, 42, 896-911. 


\section{List of Figures}

1 Vehicle "bicycle" model.

2 Vehicle in the lane.

3 "Normal driving" zone represented in a two-dimensional state space $\left(\left(y_{L}, \psi_{L}\right)\right)$.

4 The polyhedron $L(\mathbf{F})$ and the ellipsoids $\varepsilon$ and $\varepsilon_{\text {ext }}$, represented in a two-dimensional state space $\left(y_{L}, \psi_{L}\right)$.

5 (a) Video camera in the front of the vehicle. (b) DC motor on the steering column.

6 (a) Trajectories of the front wheels (solid line), predefined lane strip $\pm d= \pm 1.1 \mathrm{~m}$ (solid line), computed driving lane strip $\pm d_{\text {ext }}= \pm 1.76 \mathrm{~m}$ (dashed line), lane borders at $\pm 1.75 \mathrm{~m}$ (dash-dot line, this coincides with the previous dashed line), assistance activated on 2 (first activation rules). (b) Driver's torque (solid line), assistance torque (dash-dot line), assistance activated on 5 (first activation rules).

7 Control activation for the new switching strategy.

8 (a) Trajectories of the front wheels (solid line), predefined lane strip $\pm d= \pm 1.1 m$ (solid line), lane border (dash-dot line), assistance activated on 2 (second activation rules). (b) Driver torque (solid line), assistance torque (dash-dot line), assistance activated on 5 (second activation rules). 
9 (a) Side slip angle $\beta$ (solid line), "normal driving" value $\beta^{N}$ (dashed line), maximum computed bound $\beta^{M}$ (dash-dot line), assistance activated on 0.03 (second activation rules). (b) Yaw rate $r$ (solid line), "normal driving" value $r^{N}$ (dashed line), maximum computed bound $r^{M}$ (dash-dot line), assistance activated on 0.3 (second activation rules).

10 (a) Relative yaw angle $\psi_{L}$ (solid line), "normal driving" value $\psi_{L}^{N}$ (dashed line), maximum computed bound $\psi_{L}^{M}$ (dash-dot line), assistance activated on 0.1 (second activation rules). (b) Lateral offset $y_{L}$ (solid line), "normal driving" value $y_{L}^{N}$ (dashed line), maximum computed bound $y_{L}^{M}$ (dash-dot line), assistance activated on 1 (second activation rules).

11 (a) Steering angle $\delta_{f}$ (solid line), "normal driving" value $\delta_{f}^{N}$ (dashed line), maximum computed bound $\delta_{f}^{M}$ (dash-dot line), assistance activated on 0.04 (second activation rules). (b) Steering angle rate $\dot{\delta}_{f}$ (solid line), "normal driving" value $\dot{\delta}_{f}^{N}$ (dashed line), maximum computed bound $\dot{\delta}_{f}^{M}$ (dash-dot line), assistance activated on 0.05 (second activation rules). 
12 (a) Trajectories of the front wheels (solid line), predefined lane strip $\pm d= \pm 1.1 \mathrm{~m}$ (solid line), lane border (dash-dot line), assistance activated for the first switching strategy on 3 (solid line), assistance activated for the second switching strategy on 4 (dashed line). (b) Yaw rate $r$ (solid line), "normal driving" value $r^{N}$ (dashed line), assistance activated for the first switching strategy on 0.3 (solid line), assistance activated for the second switching strategy on 0.45 (dashed line). 


\section{List of Tables}

1 Bounds $x_{i}^{N}$ corresponding to the "normal driving" set and guaranteed bounds by control design $x_{i}^{M}$ and $\left(x_{i}^{M}\right)_{\text {new }}$ for the cases of use of the first and second switching strategy, respectively.

2 Values of the vehicle parameters. 